\title{
A Two-layered Fast Marching Path Planning Algorithm for an Unmanned Surface Vehicle Operating in a Dynamic Environment
}

\author{
Rui Song \\ Department of Mechanical Engineering \\ University College London \\ London, UK \\ r.song.11@ucl.ac.uk \\ Wenwen Liu \\ Department of Mechanical Engineering \\ University College London \\ London, UK \\ w.liu.11@ucl.ac.uk
}

\author{
Yuanchang Liu \\ Department of Mechanical Engineering \\ University College London \\ London, UK \\ yuanchang.liu.10@ucl.ac.uk \\ Richard Bucknall \\ Department of Mechanical Engineering \\ University College London \\ London, UK \\ r.bucknall@ucl.ac.uk
}

\begin{abstract}
This paper describes a modified fast marching (FM) path planning algorithm for unmanned surface vehicles (USVs). The modified FM algorithm generates a two-layered synthetic vector field to represent a dynamic environment. The synthetic vector field integrates the obstacle information and the environment information, where current and wind velocities vary in both magnitude and direction. The path planning algorithm then employs the anisotropic FM method to calculate a safe trajectory to avoid obstacles and to minimise any negative effects of the environment. The algorithm has been tested in the environment with simulated current. The resulting trajectory shows that the two-layered FM algorithm is able to deal with environmental influence satisfactory.
\end{abstract}

Keywords-marine environment; anisotropic medium; fast marching method; path planning; unmanned surface vehicle

\section{INTRODUCTION}

Unmanned surface vehicle (USV) is different to other unmanned autonomous vehicles such as unmanned ground vehicle (UGV) and unmanned aerial vehicle (UAV) because of its complex operating environment. The marine environment, especially the ocean, is always related to the nouns of uncertainty, unsafety and adjectives of complex and volatile. These properties may jeopardise USV missions en route, which should be included when developing path planning algorithms.

The path planning problem, which is fundamentally an optimisation problem, determines a trajectory subjected to various optimisation requirements. The total distance and the overall safety are usually two main concerns. In addition, the quality of a generated path such as the smoothness and continuity of the path is another aspect that need to be taken into account. It is well known that the FM based path planning

This work is supported by the ACCeSS group. The Atlantic Centre for the innovative design and Control of Small Ships (ACCeSS) is an ONR-NNRNE programme with Grant no. N0014-10-1-0652. algorithm offers benefits of improved trajectory smoothness and continuity in contrast to other algorithms such as the $\mathrm{A}^{*}$ [1], Dijisktra [2] and the genetic algorithm [3], which is why the FM has attracted special attention in recent decades for marine vehicle path planning [4][5][6][7].

Liu et al [8] successfully applied the FM method to generate a collision-free path for an USV, but a discrepancy was found between the planned path and actual trajectory when undertaking field tests. It subsequently found that the discrepancy was due to the dynamic marine environment, where current and wind velocities varied in both magnitude and direction thereby altering the actual route taken by the USV. It is therefore important to consider the influences of environmental factors when developing USV path planning algorithms, which is the aim of this paper.

In this paper, a novel two-layered FM path planning algorithm has been proposed. The algorithm uses the conventional FM method generating the base vector field (first layer) and combines the environment vector field (second layer) to form a synthetic vector field. The generated synthetic field is an anisotropic medium, where the anisotropic FM method is used to find an optimal path. The generated path has the least cost controlled by different weightings. This twolayered FM algorithm has been verified using computer simulations and proven to be effective in coping with various marine environments.

This paper is organised as follows. Section II describes the structure and the construction procedures of the synthetic vector field. Section III details the path searching algorithm based on the anisotropic FM method. Results are shown in Section IV with discussions. The paper is concluded in Section V. 


\section{SYNTHETIC VECTOR FIELD}

The focus of the two-layered FM algorithm is the construction of a synthetic vector field (VF), which combines two individual vector fields representing the configuration space and the associated environment factors. Fig. 1 shows the construction of the synthetic VF, which has a two-layer structure. The first layer is obtained by applying the conventional FM method on a configuration space to generate a base VF that represents a general path planning environment. The second layer uses the environment factors information, such as current and wind, to represent the environment VF. Details of the process for each layer are explained in the following subsections.

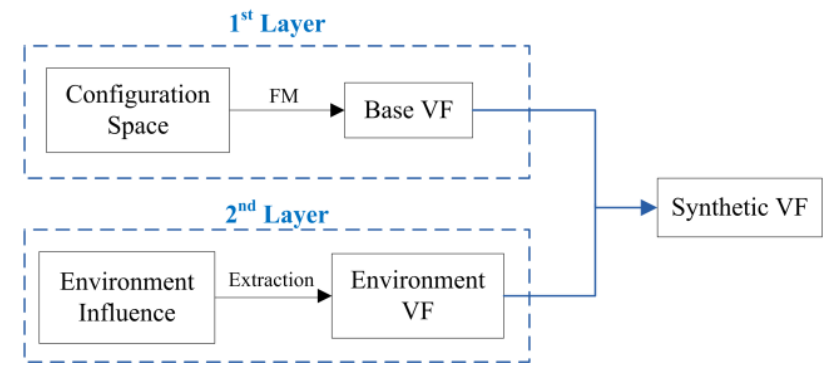

Fig. 1 Structure of the synthetic vector field.

\section{A. Fast Marching Method}

Since the fast marching (FM) has been intensively used in this paper for either generating the base VF or searching for the path, a brief introduction of the FM is first presented.

The FM was proposed with the aim of numerically solving the Eikonal equations. Both Sethian [9] and Tsitsiklis [10] provided individual algorithm explanations for the FM at the same time by using different approximation approaches. In this paper, the Tsitsiklis's method will be explained in detail as its concept has been used as the basis of the anisotropic FM, which is used later to search for the path in the synthetic $\mathrm{VF}$.

In [10], FM was proposed to solve the minimum cost problem along a trajectory. The solution to the minimum cost satisfies the Eikonal equation, which has the form of:

$$
\|\nabla U(x)\|=\tau(x) .
$$

where $U(\boldsymbol{x})$ is the cumulative cost at configuration point $\boldsymbol{x}$, and $\tau(\boldsymbol{x})$ is the weighting at $\boldsymbol{x}$. The Eikonal equation was originally develped by Hamilton and Burns for geometrical optics [10], which describes a wave front propagation scenario with the speed of the wave given as $\tau(\boldsymbol{x})$ at point $\boldsymbol{x}$. Therefore, the explanation of the FM will be given from this prospective. In this paper, the 4-geometry connection (8 neighbours in total around $\boldsymbol{x}$, see Fig. $2 \mathrm{a}$ ), is used when executing the FM method. As shown in Fig. 2(b), the wave arrival time cost at $\boldsymbol{x}(u(\boldsymbol{x}))$ is updated in form of

$$
u_{x_{i} x_{j}}(x)=\min _{t_{1}, t_{2}}\left(t_{1} u_{x_{i}}+t_{2} u_{x_{j}}+\sqrt{t_{1}^{2}+t_{2}^{2}} \cdot \tau_{x}\right),
$$

where $u_{x_{i}}$ and $u_{x_{j}}$ are the costs of $\boldsymbol{x}$ 's neighbour points $\boldsymbol{x}_{i}$ and $\boldsymbol{x}_{j}$, respectively. $\tau_{x}$ is calculated as the Euclidean distance between $\boldsymbol{x}$ and the intersection point $M . t_{1}$ and $t_{2}$ satisfy the following conditions: $t_{1}+t_{2}=1$ and $t_{1}, t_{2}>0$. Note that the travel of the wave front follows a circular cost/speed characteristic at each point $\boldsymbol{x}$. Therefore, there is no direction preference, which means that the 8 neighbour points of $\boldsymbol{x}$ have the same priority when being updated in the next time step.

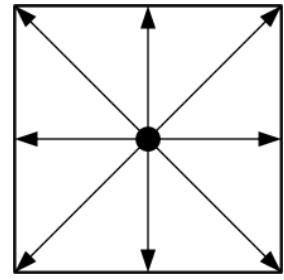

(a)

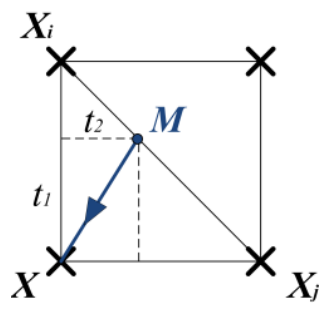

(b)
Fig. 2 (a) 4-geometry cell connection scheme, (b) the FM method updating scheme.

\section{B. Base Vector Field}

\section{1) Configuration space}

The configuration space (C-space) is the space where the path planning algorithm is run. It consists of the obstacles space $\left(C_{o b s}\right)$ and the collision-free space $\left(C_{\text {free }}\right)$. In this paper, the configuration space is obtained from the data acquisition module of the USV navigation system [8], which has an ambiguous expression of $C_{o b s}$ and $C_{\text {free }}$. Therefore, the Otsu method [11] is used to generate a black and white map to distinguish the area of $C_{o b s}$ and $C_{\text {free }}$. The black and white map is further rasterised into a grid map, which forms the configuration space.

Fig. 3(a) shows the map of the Roadford Lake, which is the raw environment. Fig. 3(b) is the generated configuration space transforming a $2 * 2 \mathrm{~km}$ to a binary map with the dimension of $500 * 500$ pixels. The $C_{o b s}$ and $C_{\text {free }}$ are represented in black and white with clear identifications. The size of each grid in the configuration space is one pixel, which is $4 \mathrm{~m}$.

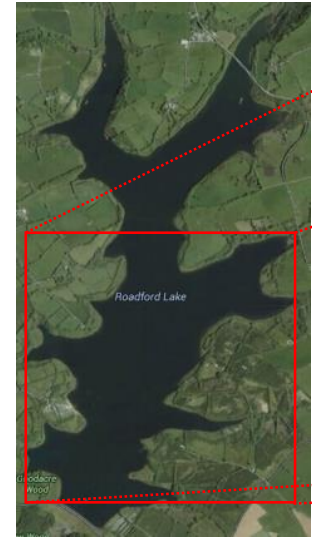

(a)

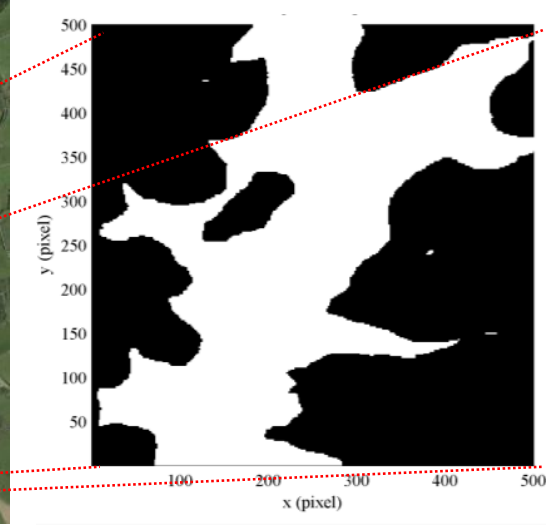

(b)
Fig. 3 (a) Map of the Roadford Lake, with dimension of 2.13*3.93 km, (b) configuration space extracted and converted from (a), the dimension is $500 * 500$ pixels. 


\section{2) The vectorisation of the configuration space}

Since the environment effect such as current is expressed in vector form, it is therefore required to vectorise the $C$-space so it may be merged with any environment influence. In this paper, a novel FM based $C$-space vectorisation approach is proposed. It consists of two steps: 1) using the FM to generate an attractive potential field and repulsive potential field, 2) calculating the gradients of two potential field to have the VFs.

In the first step, the FM method is run twice. First, the FM algorithm starts the wave propagation from USV's mission goal point to generate the attractive potential field, where the further the distance to goal point is, the higher the potential. Then, the FM is run for the second time however from all points representing the obstacle area so produces a repulsive potential field.

In terms of the second step, the attractive and repulsive potential fields are vectorised by calculating the gradients. The gradient of each potential field is calculated using forward difference and is normalised to obtain the attractive VF and the repulsive VF. In such cases, the attractive VF simulates the directions of the attractive forces pointing towards the mission goal point; and the repulsive VF provides the direction information of the repulsive forces from the obstacles.

Finally, the base VF $\left(F_{\text {base }}\right)$ is obtained as the weighted combination of two VFs as:

$$
F_{\text {base }}=\alpha_{1} F_{\text {att }}+\alpha_{2} F_{\text {rep }},
$$

where $F_{\text {att }}$ and $F_{\text {rep }}$ present the attractive and repulsive forces. $\alpha_{1}$ and $\alpha_{2}$ are corresponding risk weightings subjected to:

$$
\alpha_{1}+\alpha_{2}=1 \text {. }
$$

The values of $\alpha_{1}$ and $\alpha_{2}$ depend on different risk scenarios (see Table I) by considering the distance between the USV and the obstacles (Obs), and the distance from the USV to the goal point.

Fig. 4 shows the generation of a base VF by combining: 1) a generated attractive VF (Fig. 4a) from the star point, and 2) a repulsive VF (Fig. 4b). Fig. 4(c) is subsequently the base VF, with risk weightings of $\alpha_{1}=\alpha_{2}=0.5$. The start and goal points are marked as round and star points, and the corresponding coordinates are $(76,278)$ and $(234,349)$, respectively. Note that the magnitude of the vector at each point is assigned with the same value.

\section{Environment Vector Field}

The second layer of the synthetic VF is the environment vector field with different influence factors (such as current and wind) added together. The environment data is available from online resources such as the National Oceanography Centre (NOC)). Fig. 5(a) shows a simulated current VF $\left(F_{e n v}\right)$. Random vectors are used here to represent the situation where current is un-predictable. In this case, the magnitude and direction of velocity at each point are varying.

\section{Synthetic Vector Field}

The final synthetic map is expressed as a function of $F_{\text {base }}$ and $F_{e n v}$ with expression of

$$
F_{\text {syn }}={ }_{1} F_{\text {base }}+{ }_{2} F_{\text {env }},
$$

where $\beta_{1}$ and $\beta_{2}$ are the field weightings of base and environment vector fields with constraint that $\beta_{1}+\beta_{2}=1$. The values of $\beta_{1}$ and $\beta_{2}$ is configured and depend on the mission requirement situation, i.e. whether the USV is in risk avoidance situation or it is in safe voyage situation. Table II explains the settings of the relation of $\beta_{1}$ and $\beta_{2}$. The combined synthetic VF of Fig. 4(c) and Fig. 5(a) is shown in Fig. 5(b), with same field weightings.

TABLE I. RISK WEIGHTINGS IN DIFFERENT SCENARIOS

\begin{tabular}{lc}
\hline \multicolumn{1}{c}{ Scenarios } & Weightings \\
\hline Certain distance away from Obs, near the end point & $\alpha_{1}>\alpha_{2}$ \\
In the middle between the Obs and the end point & $\alpha_{1}=\alpha_{2}$ \\
Close to the safe area of Obs & $\alpha_{1}<\alpha_{2}$ \\
\hline
\end{tabular}

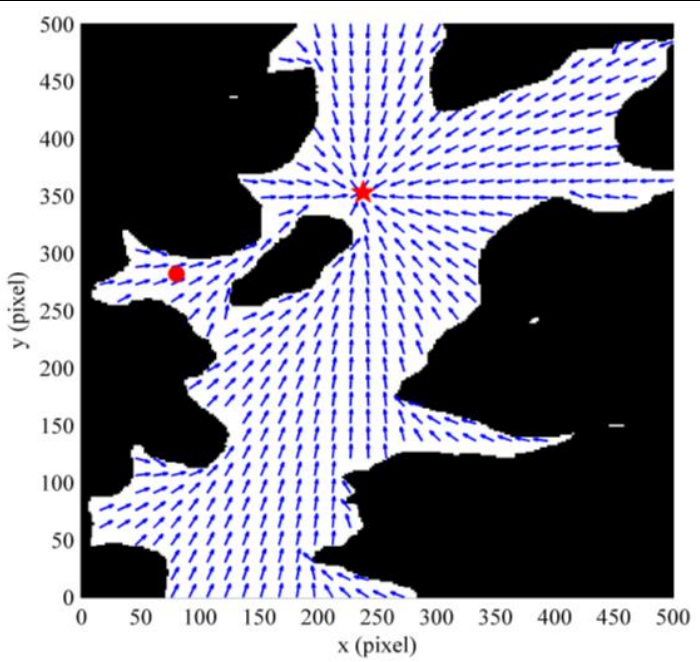

(a)

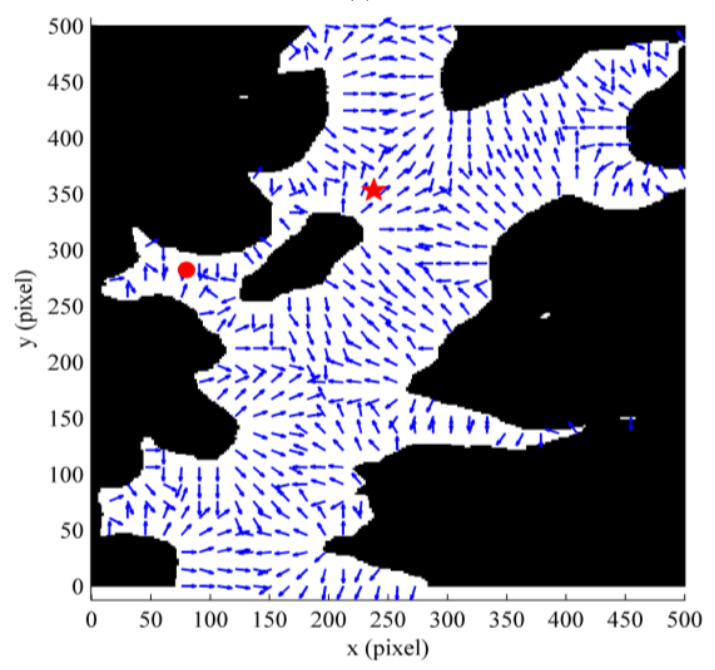

(b) 


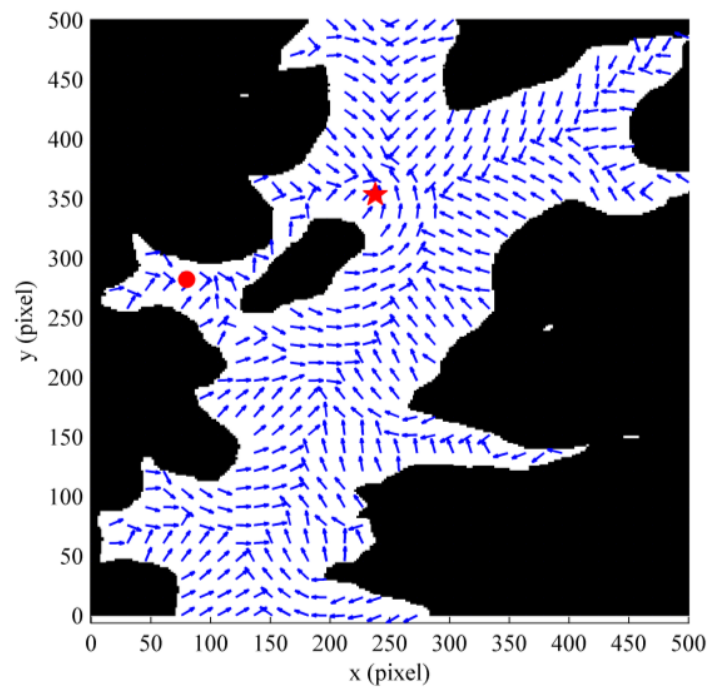

(c)

Fig. 4 (a) Attractive VF generated from the goal point (star point), (b) Repulsive VF produced from all the points of obstacle area, and (c) Base VF generated with equal risk weightings, namely $\alpha_{1}=\alpha_{2}=0.5$.

TABLE II. FIELD WEIGHTINGS IN DIFFERENT MISSION REQUIREMENTS

\begin{tabular}{lc}
\hline \multicolumn{1}{c}{ Mission requirements } & Weightings \\
\hline Collision avoidance is in priority & $\beta_{1}>\beta_{2}$ \\
In peaceful environment & $\beta_{1}=\beta_{2}$ \\
In strong current environment & $\beta_{1}<\beta_{2}$ \\
\hline
\end{tabular}

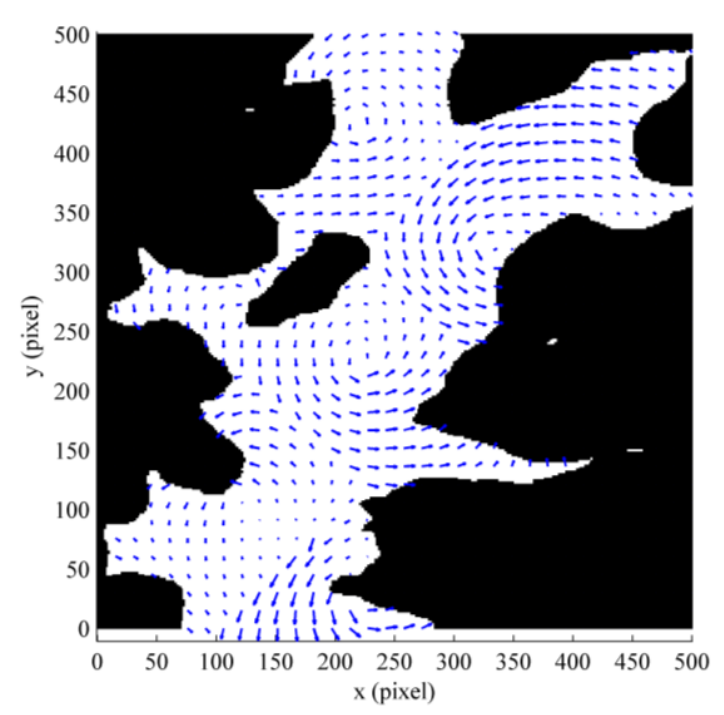

(a)

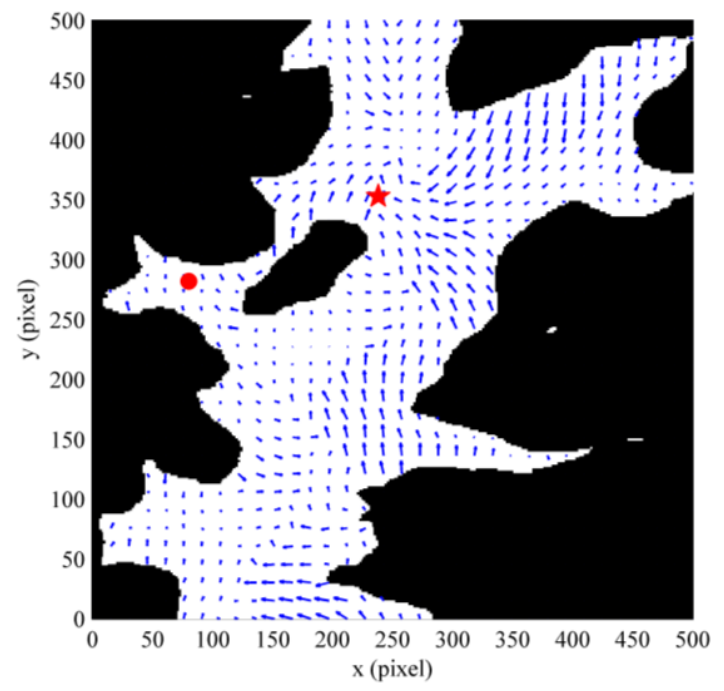

(b)

Fig. 5 (a) Current VF with varying vector magnitudes, (b) Synthetic VF, with equal field weightings, namely $\beta_{1}=\beta_{2}=0.5$.

\section{ANISOTROPIC FAST MARCHING}

It is required to take the effect of vector orientation into consideration when the path planning algorithm is performed on the synthetic VF, where any useful information is expressed in form of a vector. However, from equation (2), it can be observed that the conventional FM method only takes the distance cost into account. To integrate the orientation, the conventional FM was improved by Lin [12], to a new algorithm named as the anisotropic FM algorithm. The anisotropic FM algorithm is described in Algorithm 1. The Accepted set in Algorithm 1 is defined as the set of the configuration points, in which their $u$ value have been updated and will not be changed; the Trial set is the set of the configuration points that their $u$ values have been calculated and are going to be updated; the Far set is the set of all the other configuration points that their $u$ values have not been computed. The updating scheme of local arrival time is described in Algorithm 2, where $u_{x_{i} x_{j}}\left(x_{n}\right)$ is a temporary $u$ value of $\boldsymbol{x}_{n}$ (neighbour point) in the expression of

$$
u_{x_{i} x_{j}}\left(x_{n}\right)=\min _{t \in[0,1]}\left(t u_{x_{i}}+(1-t) u_{x_{j}}+\frac{\|\vec{\theta}(t)\|}{f(\vec{\theta}(t))}\right) \text {. }
$$

Here $\vec{\theta}(t)=\|\vec{\theta}\|(\cos \angle \vec{\theta}, \sin \angle \vec{\theta})$ is a local vector of the wave travel direction at $\boldsymbol{x}_{n}$ 's location (see in Fig. 6b). $f(\vec{\theta}(t))$ is the local speed along $\vec{\theta}(t)$, and its reciprocal is the corresponding cost denoted as $\tau(\vec{\theta}(t))$. 

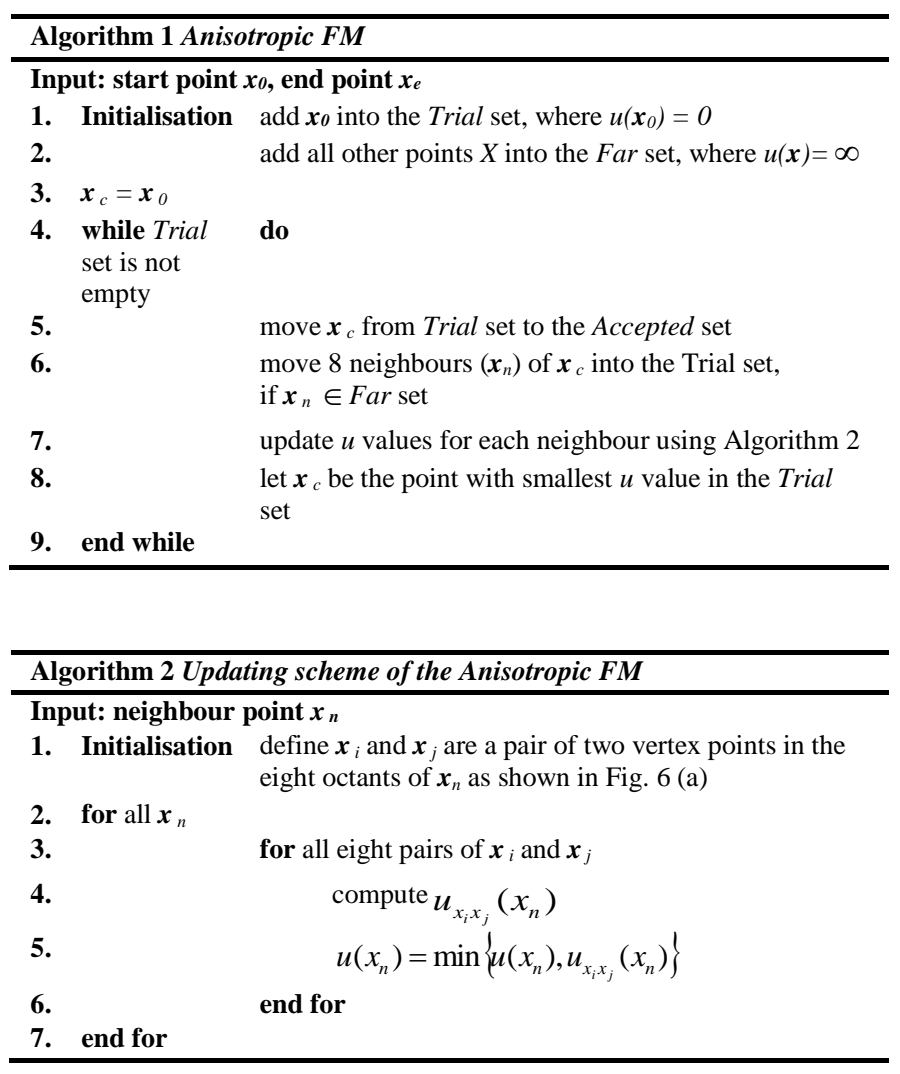

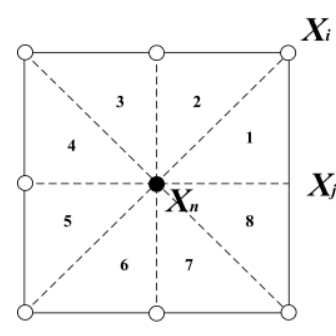

(a)

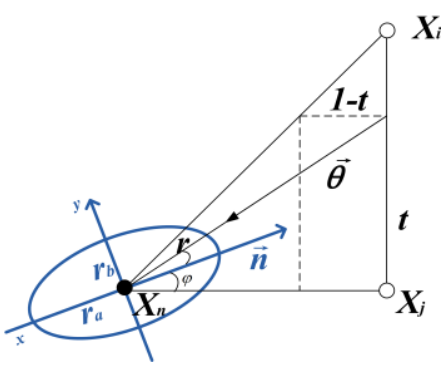

(b)
Fig. 6 (a) Eight octants definition of the anisotropic FM updating scheme, (b) Schematic of calculating arrival time at neighbor point $\boldsymbol{x}_{n}$.
Different from the conventional FM method, the local cost/speed characteristic of the anisotropic FM is no longer a circle. In this paper, the ellipse shape is used to represent the local cost/speed model, as it is easy to convert to a circle shape (conventional FM case). The direction of each vector on the generated synthetic VF is defined as the major axis of the ellipse; while its minor axis is perpendicular to the vector's direction. In this case, the wave front travels along the major axis as the propagation preferred direction. In a general case, the ellipse speed profile is described as

$$
\frac{x^{2}}{r_{a}^{2}}+\frac{y^{2}}{r_{b}^{2}}=1
$$

where $r_{a}$ and $r_{b}$ are the radii along the $x$ and $y$ axis. If the ellipse is along a direction of $\vec{n}$, and the wave front comes from the first octant (as shown in Fig. 6 (b)), then the speed along $\vec{\theta}$, denoted as $r$ is given with $x=r \cos \angle(\vec{\theta}, \vec{n})$ and $y=r \sin \angle(\vec{\theta}, \vec{n})$. Substitute the $x$ and $y$ into equation (6), we can get that

$$
r^{2}\left(\frac{(\cos \angle(\vec{\theta}, \vec{n}))^{2}}{r_{a}{ }^{2}}+\frac{(\sin \angle(\vec{\theta}, \vec{n}))^{2}}{r_{b}{ }^{2}}\right)=1 .
$$

The reciprocal of the speed $\frac{1}{r}$, which is the cost function $\tau(\vec{\theta})$, is now in the expression of

$$
\begin{gathered}
\tau(\vec{\theta})=\sqrt{\frac{(\cos \angle(\vec{\theta}, \vec{n}))^{2}}{r_{a}^{2}+\frac{(\sin \angle(\vec{\theta}, \vec{n}))^{2}}{r_{b}{ }^{2}}}} \\
=\frac{1}{\|\vec{\theta}\|}\left(\sqrt{\frac{\left(\vec{\theta}_{x} \cos \varphi\right)^{2}}{r_{a}^{2}}+\frac{\left(\vec{\theta}_{y} \sin \varphi\right)^{2}}{r_{b}{ }^{2}}}\right),
\end{gathered}
$$

where $\varphi$ is the angle between $\vec{n}$ and $x$ axis. $\vec{\theta}_{x}$ and $\vec{\theta}_{y}$ are components of $\vec{\theta}$ along $x$ and $y$ axis. Therefore, equation (5) can be rewritten as

$$
u_{x_{i} x_{j}}\left(x_{n}\right)=\min _{t \in[0,1]}\left(t u_{x_{i}}+(1-t) u_{x_{j}}+\sqrt{\frac{\left(\vec{\theta}_{x} \cos \varphi\right)^{2}}{r_{a}^{2}}+\frac{\left(\vec{\theta}_{y} \sin \varphi\right)^{2}}{r_{b}^{2}}}\right)
$$

As described in Algorithm 1, the anisotropic FM algorithm stops searching once the Trial set is empty. After this propagation process, a new potential field, named as the synthetic potential field is generated with the highest potential value ( $u$ value, the arrival time cost) at the end point $\boldsymbol{x}_{e}$, while the smallest potential is at the start point $\boldsymbol{x}_{0}$ (potential is 0 ). The minimum-cost (refer the time cost as the distance cost) path is then extracted from $\boldsymbol{x}_{e}$ to $\boldsymbol{x}_{0}$ following a gradient decent manner on this synthetic potential field.

\section{RESULTS}

The two-layered FM path planning algorithm was tested in a simulated environment that is presented in Fig. 3(b). The environment is rasterised with dimension of $500 * 500$ pixels. The performance of the anisotropic FM algorithm was first 
compared by running the conventional FM algorithm on the general path planning environment. Then the Anisotropic FM algorithm was applied on the base VF (as seen in Fig. 4c) to test its orientation guidance ability. Finally, the anisotropic FM was verified on the synthetic VF (as shown in Fig. 5b) with different field weightings. made:

In the simulations, the following assumptions have been

1) The USV is configured as a mass point, and the influence of the forces acting on the USV is regarded as being simulated to the effect of the generated vector at each grid point.

2) The simulations are in a $2 \mathrm{D}$ space, where the coordinate of each point $\boldsymbol{x}$ is in the form of $(x, y)$. All simulations have the same start and end points. The coordinates of start point is $(76,278)$, and the end point is $(234,349)$.

3) The mission goal point is set as the algorithms' start point, and the mission start point is the end point. This allows the gradient decent procedures to take place from the mission start point.

The conventional FM algorithm was run first on the environment shown in Fig. 3 (b). The generated potential field is shown in Fig. 7 (a) with Fig. 7 (b) as a rotated 3D version of Fig. 7 (a). The colour from blue to red represent different potential values from low to high. It can be observed that the end point has the lowest potential. It can also be seen from Fig. 7(a) that the wave was propagation in a circle shape, which reflects the cost/speed profile of the conventional FM. The blue line in Fig. 8 shows the generated path with the middle part staying too close to obstacles.

The anisotropic FM algorithm was then run on the base VF (see Fig. 4c). The generated potential field and path are shown in Fig. 9 and Fig. 10. Compared with the blue path in Fig. 8, it can be seen that the green path follows the vectors direction guidance with the aim of reaching the end point. In addition, the green path is not as close as the blue path to the obstacles. This is because that the green path considered the influence of the repulsive forces generated from the obstacles.

Finally, the anisotropic FM algorithm was run on the synthetic VF that takes the current influence into account. Fig. 11 shows the generated corresponding synthetic potential field. From Fig. 9 and Fig.11, it can be seen that the shapes of these two propagations are not circular. The generated path is shown in Fig.12, with similar field weightings. Comparing three paths in Fig.8 (denoted as FM_Path), Fig. 10 (Base_Path) and Fig. 12 (Syn_Path1), the Syn_Path1 looks different to the other two paths. It passes the right-hand side of the middle obstacle to get to the mission goal point. Although it takes a longer distance cost, it avoids the potential collision risk to the left land when the current has influences on the USV.

It is evident in Fig.12 that the Syn_Path1 is still too close to the middle obstacle when near to the mission end point. To address this problem, the risk weighting of the repulsive VF can be increased. Fig.13 shows the simulated path (denoted as Syn_Path2) result when $\alpha_{1}=0.1$ and $\alpha_{2}=0.9$. It can be seen that the Syn_Path2 is much safer than Syn_Path1, as it keeps a certain distance away from all obstacles.

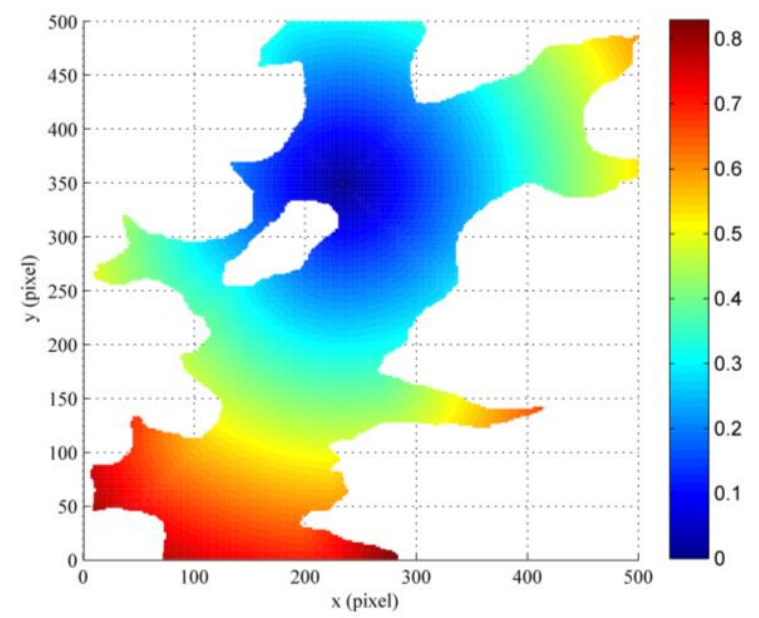

(a)

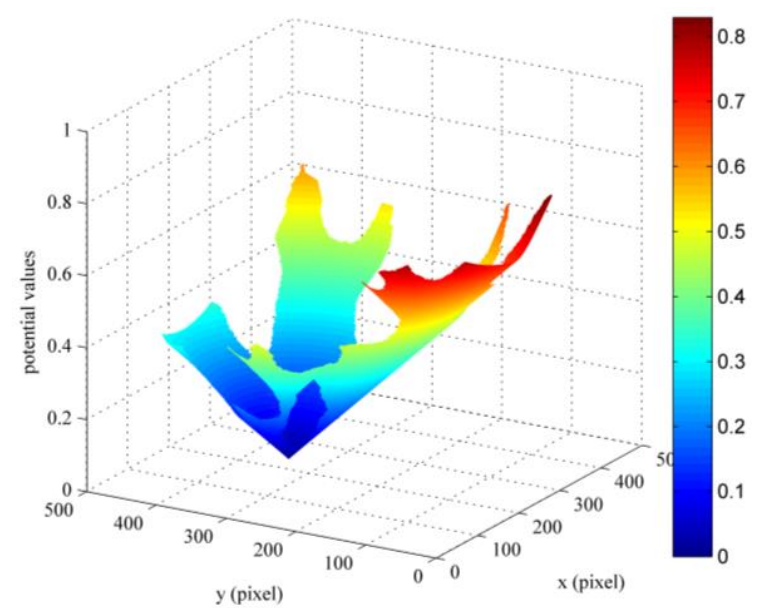

(b)

Fig. 7 Generated potential field by the conventional FM method (a) in 2D view and (b) 3D.

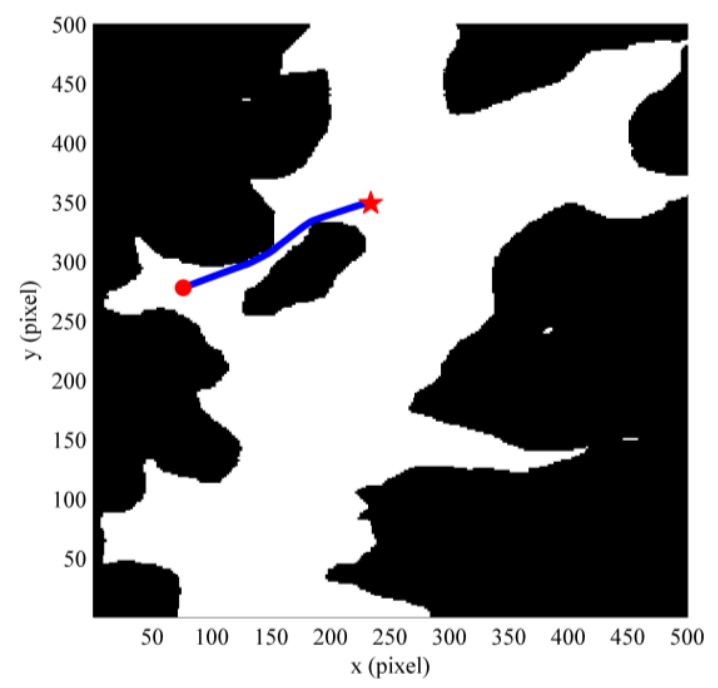

Fig. 8 Calculated path (FM_Path) by applying the conventional FM method on the configuration space. 


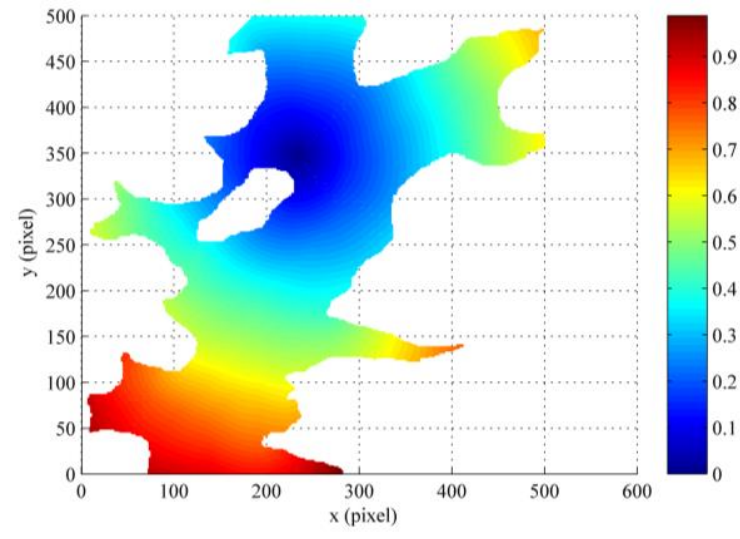

(a)

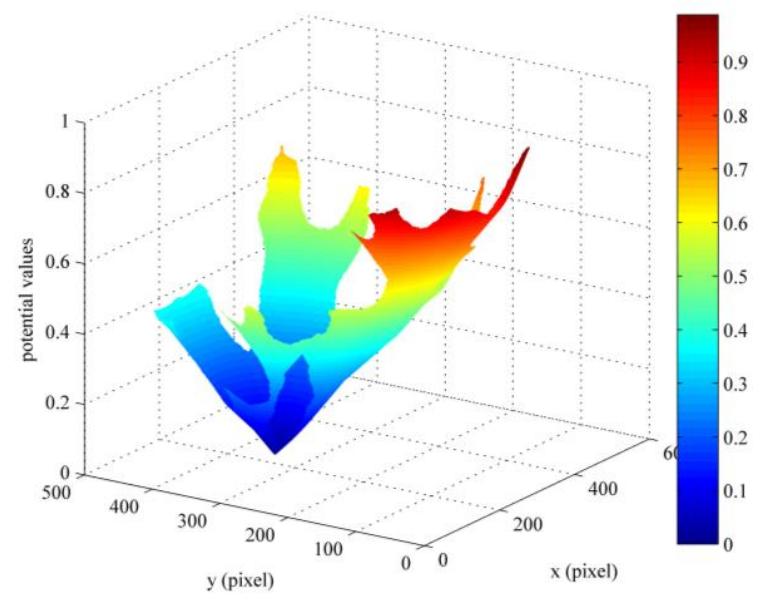

(b)

Fig. 9 Generated base potential field by the two-layered FM algorithm on the base $\mathrm{VF}$ (a) in 2D view and (b) in 3D.

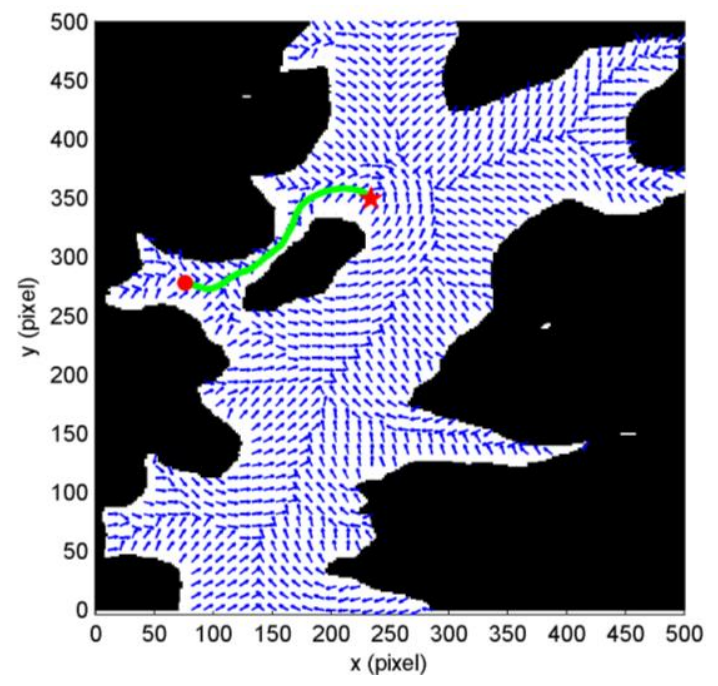

Fig. 10 Calculated path (Base_Path) by applying the two-layered FM method on the base VF, with $\alpha_{1}=\alpha_{2}=0.5$.

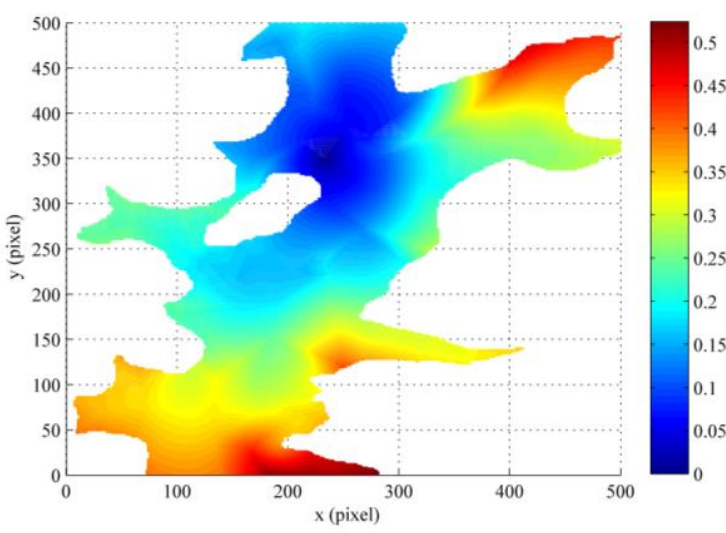

(a)

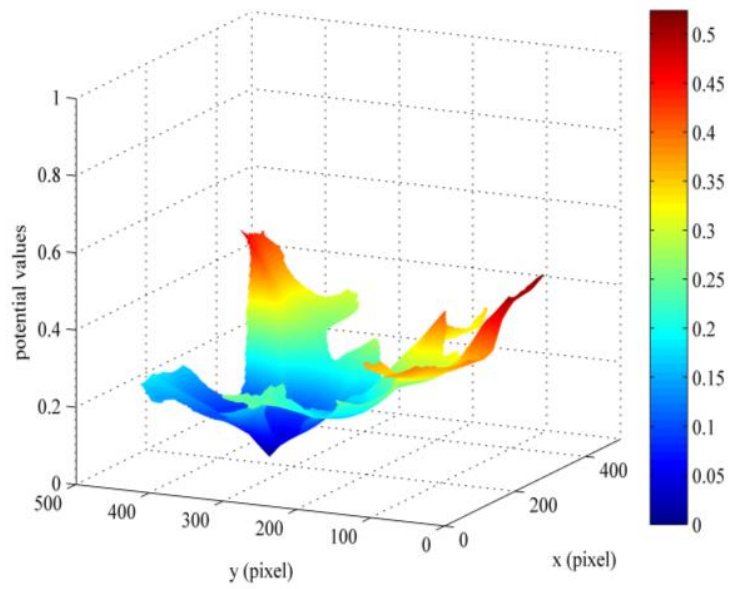

(b)

Fig. 11 Generated synthetic potential field by the two-layered FM algorithm on the synthetic VF (a) in 2D view and (b) in 3D view.

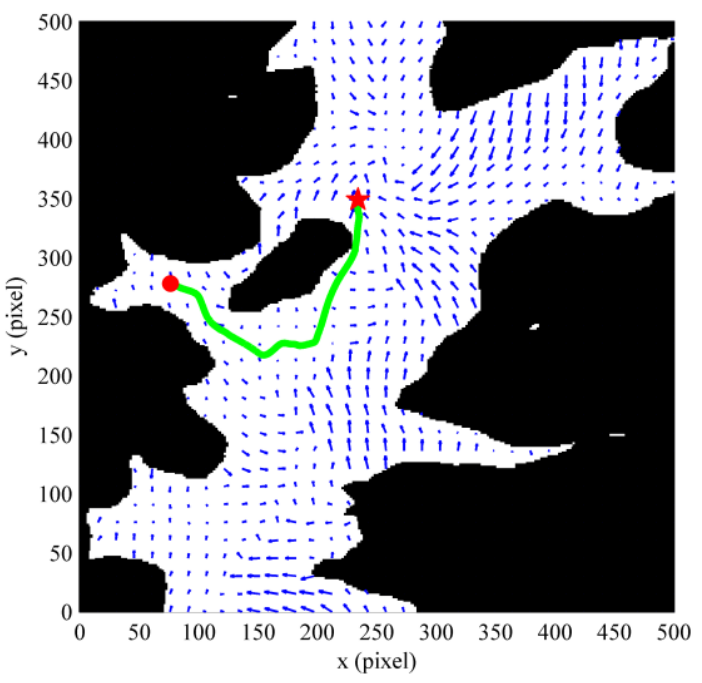

Fig. 12 Calculated path (Syn_Path1) by applying the two-layered FM algorithm on the synthetic VF, with $\alpha_{1}=\alpha_{2}=0.5$ and $\beta_{1}=\beta_{2}=0.5$. 


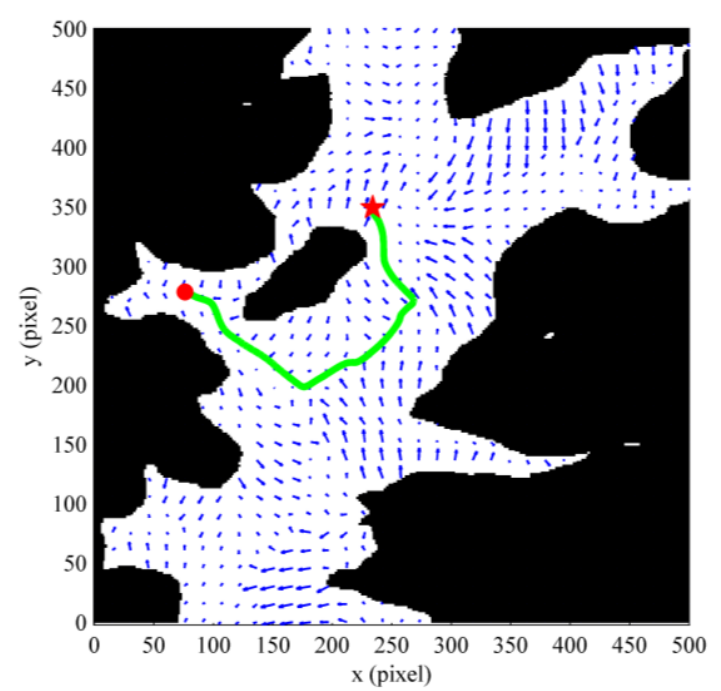

Fig. 13 Optimum path (Syn_Path2) obtained by the two-layered FM algorithm applying on the synthetic VF, with $\alpha_{1}=0.1, \alpha_{2}=0.9$ and $\beta_{1}=\beta_{2}=0.5$.

\section{CONCLUSIONS}

A novel two-layered FM path planning method has been developed to provide collision-free path for USVs. The algorithm generates a base vector field and an environment vector field combining to give a synthetic vector field, where the anisotropic FM method is used to find an optimal path. The generated path is shown to be controlled by risk and field weightings.

The effect of environmental influence was investigated by comparing the calculated paths between the conventional FM method and the proposed algorithms. It showed that employing the conventional FM method could lead to high collision risk in a dynamic environment where the obstacles are too close to the predicted path. In contrast, the proposed FM method provides a better path that keeps a safe distance away from obstacles, though it incur greater cost (distance) to reach the goal point. It has also been shown that the safety margin can be further increased by altering the risk and field weightings.

\section{REFERENCES}

[1] D. Dolgov, S. Thrun, M. Montemerlo, and J. Diebel, "Path Planning for Autonomous Vehicles in Unknown Semistructured Environments", The International Journal of Robotics Research, vol. 29, no.5, April 2010, pp. $485-501$

[2] J. L. Solka, J. C. Perry, B. R. Poellinger, and G. W. Rogers, "Fast computation of optimal paths using a parallel Dijkstra algorithm with embedded constraints", Neurocomputing, vol. 8, no.2, 1995, pp. 195212.

[3] C. K. Tam, and R. Bucknall, "Path- planning algorithm for ships in close- range encounters", Official Journal of the Japan Society of Naval Architects and Ocean Engineers, vol. 15, no. 4, 2010, pp. 395-407.

[4] C. Petres, Y. Pailhas, P. Patron, Y. Petillot, J. Evans, and D. Lane, "Path planning for autonomous underwater vehicles", IEEE Trans. Robot., vol. 23, no. 2, 2007, pp. 331-41.

[5] B. Rhoads, I. Mezić, and A. C. Poje, "Minimum time heading control of underpowered vehicles in time-varying ocean currents", Ocean Engineering, vol. 66, 2013, pp. 12-31.
[6] B. Xu, D. J. Stilwell, and A. J. Kurdila, "Fast Path Re- planning Based on Fast Marching and Level Sets”, J. Intell. Robot. Syst., vol. 71, no. 34, 2013, pp. 303-17.

[7] Y. Liu, and R. Bucknall, "Path planning algorithm for unmanned surface vehicle formations in a practical maritime environment", Ocean Engineering, vol. 97, 2015, pp. 126-44

[8] Y. Liu, R. Song, W. Liu, and R. Bucknall, "Autonomous Navigation System for Unmanned Surface Vehicles", 13th Computer Applications and Information Technology in the Maritime Industries, Redworth, UK 2014.

[9] J. A. Sethian, "Level Set Methods and Fast Marching Methods", Cambridge University Press, Cambridge, Mass, USA, 2002.

[10] J. N. Tsitsiklis, "Efficient algorithms for globally optimal trajectories", IEEE Transactions on Automatic Control, vol. 40, no. 9, 1995, pp. 15281538.

[11] R. Song, "Path Planning and Bi-directional Communication for Unmanned Surface Vehicle". MIDAS Technical Report, MIDAS MSE.2014.TR.011, 2014.

[12] L. Qingfen, "Enhancement, extraction, and visualization of 3D volumedata", Ph.D.thesis, Linko ping University, Linko ping, Sweden, 2003. 